\title{
Hybrid Method for Solving New Pose Estimation Equation System ${ }^{1)}$
}

\author{
Greg Reid, Jianliang Tang, Jianping Yu and Lihong Zhi \\ Dept. of Applied Mathematics, University of Western Ontario \\ London, Canada N6A 5B7 \\ reid@uwo.ca \\ Department of Mathematics, College of Science, Shenzhen University \\ Shenzhen 518060, P.R.china \\ Key Laboratory of Mathematics Mechanization \\ Institute of Systems Science, AMSS, Academia Sinica, Beijing 100080, China \\ lzhi@mmrc.iss.ac.cn
}

\begin{abstract}
Camera pose estimation is the problem of determining the position and orientation of an internally calibrated camera from known 3D reference points and their images. We introduce a new polynomial equation system for 4-point pose estimation and apply our symbolic-numeric method to solve it stably and efficiently. In particular, our algorithm can also recognize the points near critical configurations and deal these near critical cases carefully. Numerical experiments are given to show the performance of the hybrid algorithm.
\end{abstract}

\section{Introduction}

Given a set of correspondences between 3D reference points and their images, 4-point pose estimation consists of determining the position and orientation of the camera with respect to four known reference points. It is a classical and common problem in computer vision and photogrammetry and has been studied in the past $[1,6,8,11,20,2,23]$.

The well-known polynomial system (1) corresponding to the 4-point pose estimation generically has a unique positive solution. It can be found successfully by linear algorithms proposed in $[20,2,23]$. But there are certain degenerate cases for which no unique solution is possible. These critical configurations are known precisely and include the following notable degenerate case: a 3D line and a circle in an orthogonal plane touching the line. In [2] an algorithm is presented that solves the problem including the critical configurations, but the relative error and failure rate (backward error) are significantly higher than one would like. In [23], the authors present a new linear algorithm which works well even in the degenerate cases. However, the matrices are much larger $70 \times 90$ compared with $24 \times 24$ matrices used in $[2]$.

In this paper, we introduce a new variable and transform the polynomial system for 4-point pose estimation to a new system with only five equations and three variables. Our

\footnotetext{
${ }^{1}$ Supported by a National Key Basic Research Project of China and Chinese National Science Foundation under Grant 10401035 and Reids Canadian NSERC Grant
} 
symbolic-numeric method can also be applied to the new system and find solutions in general or critical cases. The matrices we used in the general or critical cases are of size $20 \times 20$; while in near critical cases, we are using a matrix of size $35 \times 50$ in order to recover the sensitive unique root.

The rest of the paper is organized as follows. In Section 2, we introduce the basic geometry of the 4-point pose estimation problem. A new system of equations is introduced. In Section 3, we briefly review the symbolic-numeric method for polynomial system solving. Then, we illustrate how to apply this method to solve the polynomial system corresponding to the critical or near critical cases. In Section 4, the simulated experimental results are given. Some conclusions are given in Section 5.

\section{Geometry of camera pose from four points}

In the following, we briefly introduce the geometry of camera pose from four points. Let $C$ be the calibrated camera center, and $P_{1}, P_{2}, P_{3}, P_{4}$ be the reference points (see Fig. 1). Let $c_{12}=2 \cos \angle\left(P_{1} C P_{2}\right), c_{13}=2 \cos \angle\left(P_{1} C P_{3}\right), c_{14}=2 \cos \angle\left(P_{1} C P_{4}\right), c_{23}=2 \cos \angle\left(P_{2} C P_{3}\right)$, $c_{24}=2 \cos \angle\left(P_{2} C P_{4}\right), c_{34}=2 \cos \angle\left(P_{3} C P_{4}\right)$.

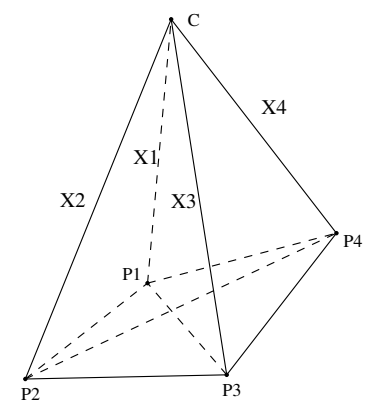

Fig. 1. The 4-point pose estimation problem

From triangles $C P_{1} P_{2}, C P_{1} P_{3}, C P_{2} P_{3}, C P_{1} P_{4}, C P_{2} P_{4}$ and $C P_{3} P_{4}$, we obtain the 4-point pose estimation equation system:

$$
\left\{\begin{array}{c}
X_{1}^{2}+X_{2}^{2}-c_{12} X_{1} X_{2}-\left|P_{1} P_{2}\right|^{2}=0 \\
X_{1}^{2}+X_{3}^{2}-c_{13} X_{1} X_{3}-\left|P_{1} P_{3}\right|^{2}=0 \\
X_{2}^{2}+X_{3}^{2}-c_{23} X_{2} X_{3}-\left|P_{2} P_{3}\right|^{2}=0 \\
X_{2}^{2}+X_{4}^{2}-c_{24} X_{2} X_{4}-\left|P_{2} P_{4}\right|^{2}=0 \\
X_{3}^{2}+X_{4}^{2}-c_{34} X_{3} X_{4}-\left|P_{3} P_{4}\right|^{2}=0 \\
X_{1}^{2}+X_{4}^{2}-c_{14} X_{1} X_{4}-\left|P_{1} P_{4}\right|^{2}=0
\end{array}\right.
$$

We are only interested in finding the positive solutions for $X_{1}, X_{2}, X_{3}, X_{4}$. Since $X_{4}=$ $\left|P_{4} C\right|$ is positive, we may make the following variable changes. Let

$$
\begin{aligned}
X_{1} & =x_{1} X_{4}, X_{2}=x_{2} X_{4}, X_{3}=x_{3} X_{4}, \\
\left|P_{1} P_{4}\right| & =\sqrt{w} X_{4},\left|P_{1} P_{2}\right|=\sqrt{a w} X_{4},\left|P_{1} P_{3}\right|=\sqrt{b w} X_{4}, \\
\left|P_{2} P_{3}\right| & =\sqrt{c w} X_{4},\left|P_{2} P_{4}\right|=\sqrt{d w} X_{4},\left|P_{3} P_{4}\right|=\sqrt{e w} X_{4} .
\end{aligned}
$$


Equation system (1) become the following equivalent equation system:

$$
\left\{\begin{array}{l}
x_{1}^{2}+x_{2}^{2}-c_{12} x_{1} x_{2}-a w=0 \\
x_{1}^{2}+x_{3}^{2}-c_{13} x_{1} x_{3}-b w=0 \\
x_{2}^{2}+x_{3}^{2}-c_{23} x_{2} x_{3}-c w=0 \\
x_{2}^{2}+1-c_{24} x_{2}-d w=0 \\
x_{3}^{2}+1-c_{34} x_{3}-e w=0 \\
x_{1}^{2}+1-c_{14} x_{1}-w=0
\end{array}\right.
$$

From $x_{1}^{2}+1-c_{14} x_{1}-w=0$ and $\left|c_{14}\right|<2\left(c_{14}=2 \cos \angle\left(P_{1} C P_{4}\right)\right)$, we have

$$
w=\left(\left|P_{1} P_{4}\right| / X_{4}\right)^{2}=x_{1}^{2}+1-c_{14} x_{1}=\left(x_{1}-c_{14} / 2\right)^{2}+1-c_{14}^{2} / 4>0 .
$$

$X_{4}$ can be uniquely determined by $X_{4}=\left|P_{1} P_{4}\right| / \sqrt{w}$ and the equivalent correspondence is:

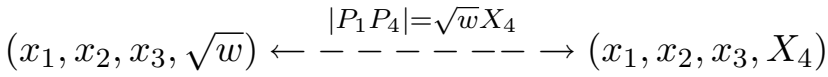

$$
\begin{aligned}
& \stackrel{X_{1}=x_{1} X_{4}, X_{2}=x_{2} X_{4}, X_{3}=x_{3} X_{4}}{\leftarrow----------\longrightarrow}\left(X_{1}, X_{2}, X_{3}, X_{4}\right)
\end{aligned}
$$

Substituting $w$ into above equation system, we have the following equivalent equation system:

$$
\left\{\begin{array}{l}
(1-a) x_{1}^{2}+x_{2}^{2}-c_{12} x_{1} x_{2}-a\left(1-c_{14} x_{1}\right)=0 \\
(1-b) x_{1}^{2}+x_{3}^{2}-c_{13} x_{1} x_{3}-b\left(1-c_{14} x_{1}\right)=0 \\
(1-c) x_{2}^{2}+x_{3}^{2}-c_{23} x_{2} x_{3}-c\left(1-c_{14} x_{1}\right)=0 \\
(1-d) x_{2}^{2}+1-c_{24} x_{2}-d\left(1-c_{14} x_{1}\right)=0 \\
(1-e) x_{3}^{2}+1-c_{34} x_{3}-e\left(1-c_{14} x_{1}\right)=0 .
\end{array}\right.
$$

The equation system (4) is simpler than the original system (1), and from the positive solution $x_{i}$ we can get the coordinates $X_{i}$ according to the equivalent correspondence. The recovered camera-point distances $X_{i}$ are used to estimate the coordinates of the 3D reference points in a camera-centered 3D frame: $\bar{P}_{i}=X_{i} K^{-1} U_{i}$ (see [20]). The final step is the absolute orientation determination [21]. The determination of the translation and the scale follow immediately from the estimation of the rotation.

The system (4) is still an overdetermined polynomial system of five equations in 3 variables. The parameters $c_{i j}(1 \leq i, j \leq 4)$ and $a, b, c, d, e$ are data of limited accuracy. It is still very difficult to use Gröbner basis algorithms [4] or Ritt-Wu's characteristic algorithms $[29,31]$ to solve such approximate overdetermined polynomial systems. In the following, we briefly introduce our new developed complete linear method [23] for solving such system stably.

\section{Linear methods for pose determination from 4 points}

Consider a general polynomial system $S$ in $x_{1}, \ldots, x_{n}$ of degree $q$ and its corresponding vector of monomials of degree less than or equal to $q$. The system can be written as

$$
M_{0} \cdot\left[x_{1}^{q}, x_{1}^{q-1} x_{2}, \ldots, x_{n}^{2}, x_{1}, \ldots, x_{n}, 1\right]^{T}=[0,0, \ldots, 0,0, \ldots, 0,0]^{T}
$$


in terms of its coefficient matrix $M_{0}$. Here and hereafter, $[\ldots]^{T}$ means the transposition. Further, $\left[\xi_{1}, \xi_{2}, \ldots, \xi_{n}\right]$ is one of the solutions of the polynomial system, if and only if

$$
\left[\xi_{1}^{q}, \xi_{1}^{q-1} \xi_{2}, \ldots, \xi_{n}^{2}, \xi_{1}, \ldots, \xi_{n}, 1\right]^{T}
$$

is a null vector of the coefficient matrix $M_{0}$.

Since the number of monomials is usually bigger than the number of polynomials, the dimension of the null space can be big. The aim of completion methods, such as ours and those based on Gröbner bases and others $[15,12,5,14,17,18,16,25,28]$, is to include additional polynomials belonging to the ideal generated by $S$, to reduce the dimension to its minima.

The bijection

$$
\phi: x_{i} \leftrightarrow \frac{\partial}{\partial x_{i}}, \quad 1 \leq i \leq n,
$$

maps the system $S$ to an equivalent system of linear homogeneous PDEs denoted by $R$. Jet space approaches are concerned with the study of the jet variety

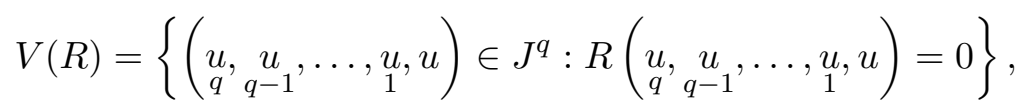

where $u$ denotes the formal jet coordinates corresponding to derivatives of order exactly $j$.

A single prolongation of a system $R$ of order $q$ consists of augmenting the system with all possible derivatives of its equations, so that the resulting augmented systems, denoted by $D R$, has order $q+1$. Under the bijection $\phi$, the equivalent operation for polynomial systems is to multiply by monomials, so that the resulting augmented system has degree $q+1$.

A single geometric projection is defined as

$$
E(R):=\left\{(\underset{q-1}{u}, \ldots, u, u) \in J^{q-1}: \exists \underset{q}{u}, R(\underset{q}{u} \underset{q-1}{u}, \ldots, \underset{1}{u}, u)=0\right\} .
$$

The projection operator $E$ maps a point in $J^{q}$ to one in $J^{q-1}$ by simply removing the jet variables of order $q$ (i.e. eliminating $\underset{q}{u}$ ). For polynomial systems of degree $q$, by the bijection $\phi$, the projection is equivalent to eliminating the monomials of the highest degree $q$. To numerically implement an approximate involutive form method, we proposed in $[30,23]$ a numeric projection operator $\hat{E}$ based on singular value decomposition.

By the famous Cartan-Kuranishi Theorem [10, 19, 27], after application of a finite number of prolongations and projections, the algorithm above terminates with an involutive or an inconsistent system.

Suppose that $R$ is involutive at prolonged order $k$ and projected order $l$, and by the bijection $\phi$ has corresponding system of polynomials $S$. Then the dimension of $\hat{E}^{l}\left(D^{k} R\right)$ allows us to determine the number of approximate solutions of $S$ up to multiplicity. In particular these solutions approximately generate the null space of $\hat{E}^{l}\left(D^{k} R\right)$. We can compute eigenvalues and eigenvectors to find these solutions. It should be noticed that the above symbolic prolongation and numeric projection method works only for solving the polynomial systems with finite number of solutions.

The following example corresponds to the third singular case as pointed in $[2,23]$. In the example the coordinate of the camera point is $(1,1,1)$, and the coordinates of the four control 
points are $(-1,1,0),(-1,-1,0),(1,-1,0)$ and $(1,1,0)$ respectively. The corresponding 4point pose estimation equation system is:

$$
\left\{\begin{array}{l}
p_{1}:=x_{2}^{2}-2.0 x_{1}^{2}-0.666667 x_{2}+1.78885 x_{1}-1.0 \\
p_{2}:=x_{3}^{2}-x_{1}^{2}-0.894427 x_{3}+0.894427 x_{1} \\
p_{3}:=x_{2}^{2}-1.49071 x_{1} x_{2}+0.894427 x_{1}-1.0 \\
p_{4}:=-x_{1}^{2}+x_{3}^{2}-0.4 x_{1} x_{3}+1.78885 x_{1}-2.0 \\
p_{5}:=x_{2}{ }^{2}+x_{3}^{2}-1.49071 x_{2} x_{3}-x_{1}^{2}+0.894427 x_{1}-1.0
\end{array}\right.
$$

We show how our symbolic-numeric method can be used to solve (10). Under the bijection $\phi: x_{i} \leftrightarrow \frac{\partial}{\partial x_{i}}$ where $i=1,2,3$, the system is equivalent to the PDE system $R$ :

$$
\left\{\begin{aligned}
\phi\left(p_{1}\right) u & :=\frac{\partial^{2} u}{\partial x^{2}}-2.0 \frac{\partial^{2} u}{\partial x_{1}{ }^{2}}-0.666667 \frac{\partial^{2} u}{\partial x_{2}}+1.78885 \frac{\partial u}{\partial x_{1}}-1.0 u \\
\phi\left(p_{2}\right) u & :=\frac{\partial^{2} u}{\partial x^{2}}-\frac{\partial^{2} u}{\partial x_{1}{ }^{2}}-0.894427 \frac{\partial u}{\partial x_{3}}+0.894427 \frac{\partial u}{\partial x_{1}} \\
\phi\left(p_{3}\right) u & :=\frac{\partial^{2} u}{\partial x_{2}{ }^{2}}-1.49071 \frac{\partial^{2} u}{\partial x_{1} \partial x_{2}}+0.894427 \frac{\partial u}{\partial x_{1}}-1.0 u \\
\phi\left(p_{4}\right) u & :=-\frac{\partial^{2} u}{\partial x_{1}{ }^{2}}+\frac{\partial^{2} u}{\partial x_{3}{ }^{2}}-0.4 \frac{\partial^{2} u}{\partial x_{1} \partial x_{3}}+1.78885 \frac{\partial u}{\partial x_{1}}-2.0 u \\
\phi\left(p_{5}\right) u & :=\frac{\partial^{2} u}{\partial x_{2}{ }^{2}}+\frac{\partial^{2} u}{\partial x_{3}{ }^{2}}-1.49071 \frac{\partial^{2} u}{\partial x_{2} \partial x_{3}}-\frac{\partial^{2} u}{\partial x_{1}{ }^{2}}+0.894427 \frac{\partial u}{\partial x_{1}}-1.0 u .
\end{aligned}\right.
$$

Applying the symbolic-numeric completion method to $R$ with tolerance $10^{-9}$, we obtain the table of dimensions below:

Table 1: $\operatorname{dim}\left(\hat{\mathbf{E}}^{\mathrm{l}} \mathbf{D}^{\mathrm{k}} \mathbf{R}\right)$ for $(11)$

\begin{tabular}{|l|ccccc}
\hline & $k=0$ & $k=1$ & $k=2$ & $k=3$ & $k=4$ \\
\hline$l=0$ & 5 & 2 & 2 & 2 & 2 \\
$l=1$ & 4 & 2 & 2 & 2 & 2 \\
$l=2$ & 1 & 2 & 2 & 2 & 2 \\
$l=3$ & & 1 & 2 & 2 & 2 \\
$l=4$ & & & 1 & 2 & 2 \\
$l=5$ & & & & 1 & 2 \\
$l=6$ & & & & & 1
\end{tabular}

We seek the smallest $k$ such that there exists an $l=0, \ldots, k$ with $\hat{E}^{l} D^{k} R$ approximately involutive. Passing the approximate projected elimination test amounts to test looking in the table for the first column with an equal entry in the next column on the downwards sloping diagonal (with both entries being on or above the main diagonal $k=l$ ). This first occurs for $k=1$ and $l=0,1,2$.

Applying the approximate version of the projected involutive symbol test to the example, shows that it is passed for $k=1, l=0$, and $l=1$, so we choose the largest $l(l=1)$, yielding $\hat{E} D R$ as the sought after approximately involutive system.

The involutive system has $\operatorname{dim}(\hat{E} D R)=2$ and so by the bijection the polynomial system (10) has 2 solutions up to multiplicity. In the following, we apply an eigenvalue method to solve (10).

1. Compute an approximate basis of the null space of $D R$, denoted by a $20 \times 2$ matrix $B$. Since $\operatorname{dim}(D R)=\operatorname{dim}(\hat{E} D R)=\operatorname{dim}\left(\hat{E}^{2} D R\right)=2$, the $4 \times 2$ submatrix $B_{1}$ and $10 \times 2$ 
submatrix $B_{2}$ of $B$ by deleting entries corresponding to the second and third degree monomials are bases of null spaces of $\hat{E}^{2} D R$ and $\hat{E} D R$ respectively.

2. Consider the set of all monomials of degree less than or equal to 1 :

$$
\mathcal{N}=\left[x_{1}, x_{2}, x_{3}, 1\right] .
$$

For numerical stability, we compute the singular value decomposition of $B_{1}$

$$
U, S, V:=\operatorname{SingularValues}\left(B_{1}\right) \text {. }
$$

The first two columns of $U$ form the $2 \times 4$ matrix $U_{s}$, and guarantee a stable linear polynomial set $\mathcal{N}_{p}=U_{s}^{T} \cdot \mathcal{N}^{T}$ for computing multiplication matrices.

3. The multiplication matrix of $x_{i}$ with respect to $\mathcal{N}_{p}$ can be formed as

$$
M_{x_{i}}=U_{s}^{T} \cdot B_{x_{i}} \cdot V^{T} \cdot S_{i}
$$

where $B_{x_{1}}, B_{x_{2}}, B_{x_{3}}$ are the $[1,2,3,7],[2,4,5,8]$ and $[3,5,6,9]$ rows of $B_{2}$ respectively, and $S_{i}$ is a diagonal matrix with elements which are inversions of the first two elements of $S: 1.95588,111.524$.

4. The coordinates $x_{i}$ of the double root can be found as the average of the eigenvalues of $M_{x_{i}}$ for $i=1,2,3$ :

$$
x_{1}=2.23607, x_{2}=3.0, x_{3}=2.23607 \text {. }
$$

Substituting the solution (12) into (10), we find $\left|p_{i}\left(\xi_{1}, \xi_{2}, \xi_{3}\right)\right|<0.42 \cdot 10^{-7}$ for $i=$ $1,2, \ldots, 5$. If one substitutes the positive solution (12) to the Jacobian matrix

$$
\left[\begin{array}{ccc}
\frac{\partial p_{1}}{\partial x_{1}} & \frac{\partial p_{1}}{\partial x_{2}} & \frac{\partial p_{1}}{\partial x_{3}} \\
\frac{\partial p_{2}}{\partial x_{1}} & \frac{\partial p_{2}}{\partial x_{2}} & \frac{\partial p_{2}}{\partial x_{3}} \\
\vdots & \vdots & \vdots \\
\frac{\partial p_{5}}{\partial x_{1}} & \frac{\partial p_{5}}{\partial x_{2}} & \frac{\partial p_{5}}{\partial x_{3}}
\end{array}\right]
$$

then the singular values of the transpose of the Jacobian matrix are

$$
11.8865,5.42001,0.109804 \cdot 10^{-8} \text {. }
$$

The Jacobian matrix is near singular. This tells us that the solution is quite unstable for any small perturbations. Suppose we perturb (10) by errors of order $10^{-6}$, the number of solutions read from the dimension table will generally become 1 .

In general, we obtain the following table: 
Table 2: $\operatorname{dim}\left(\hat{\mathbf{E}}^{\mathrm{l}} \mathbf{D}^{\mathrm{k}} \mathbf{R}\right)$ for near critical case

\begin{tabular}{|c|ccccc}
\hline & $k=0$ & $k=1$ & $k=2$ & $k=3$ & $k=4$ \\
\hline$l=0$ & 5 & 2 & 1 & 1 & 1 \\
$l=1$ & 4 & 2 & 1 & 1 & 1 \\
$l=2$ & 1 & 2 & 1 & 1 & 1 \\
$l=3$ & & 1 & 1 & 1 & 1 \\
$l=4$ & & & 1 & 1 & 1 \\
$l=5$ & & & & 1 & 1 \\
$l=6$ & & & & & 1
\end{tabular}

Applying the projected elimination and involutive symbol tests shows that $\hat{E}^{2} D^{2} R$ is approximately involutive. The computed positive root has backward error of order $10^{-6} \sim$ $10^{-9}$ in general.

In order to compare the difference between general cases, critical cases and near critical cases, in the below, we also show the dimension table corresponding to the general cases.

Table 3: $\operatorname{dim}\left(\hat{\mathbf{E}}^{\mathrm{l}} \mathbf{D}^{\mathrm{k}} \mathbf{R}\right)$ for general case

\begin{tabular}{|l|ccccc}
\hline & $k=0$ & $k=1$ & $k=2$ & $k=3$ & $k=4$ \\
\hline$l=0$ & 5 & 1 & 1 & 1 & 1 \\
$l=1$ & 4 & 1 & 1 & 1 & 1 \\
$l=2$ & 1 & 1 & 1 & 1 & 1 \\
$l=3$ & & 1 & 1 & 1 & 1 \\
$l=4$ & & & 1 & 1 & 1 \\
$l=5$ & & & & 1 & 1 \\
$l=6$ & & & & & 1
\end{tabular}

From the three different dimension tables, it is easy to deduce the following conclusions. Firstly, in the general case, the unique solution can be recovered from the null vector of the $20 \times 20$ matrix generated by $p_{i}, x_{i} p_{j}$ for $i, j=1,2,3$. Secondly, if the four points are on the critical configuration, we have to deal it with eigenvalue method after forming the multiplication matrix with respect to $x_{1}, x_{2}, x_{3}$ separately. Finally, if the points are near the critical configuration, then the solution should be found stably from the null vector of the $35 \times 50$ matrix generated by $p_{i}, x_{i} p_{j}, x_{i} x_{j} p_{k}$ for $i, j, k=1,2,3$. The main reason is due to that the dimension of the null space of the $20 \times 20$ matrix is two from table 2 in near degenerate cases.

\section{Experimental Results}

Based on the linear symbolic-numeric method, we may have the following algorithm for the 4-point pose estimation problem:

- Compute the $c_{i j}$ from the image points and the camera calibration matrix $K$.

- Compute the inter-point distances $\left|P_{i} P_{j}\right|$ from the reference points.

- Compute the solution $x_{1}, x_{2}, x_{3}$ of the polynomial system (4) using the symbolicnumeric method [23]. 
- Recover the camera-point distances $X_{1}, X_{2}, X_{3}, X_{4}$ from the equivalence correspondence (3).

- Estimate the coordinates of the 3D reference points in a camera-centered 3D frame: $\bar{P}_{i}=X_{i} K^{-1} U_{i}$.

- Compute the camera rotation and translation using the absolute orientation $[9,20,21]$.

We first demonstrate the accuracy and stability of hybrid algorithm for the generic cases. Then, we also check our algorithm for points near the critical configurations. The following experiments are done with Maple 8 in the default setting of digits (Digits=10).

The first experiment is to show the accuracy and stability of the algorithm for the general 4-point pose estimation. The optical center is located at the origin and the matrix of camera's intrinsic parameters is assumed to be the identity matrix. At each trial, four noncoplanar control points are generated at random within a cube centered at $(0,0,50)$ and of dimension $60 \times 60 \times 60$. The orientation Euler angles of the camera are positioned randomly. The control points are projected onto an image plane using the camera pose and internal parameters. We carry out one hundred trials and generate 100 sets of control points randomly for each trial. For a set of solutions, we substitute them into (1) and check the backward error. The backward error of the experimental results is generally less than $10^{-8}$.

We check the stability of the algorithm. The relative error of the estimated translation $t_{i}$ w.r.t. the true $t$ is measured by $2\left|t_{i}-t\right| /\left(\left|t_{i}\right|+|t|\right)$. The relative error of the estimated rotation $R_{i}$ w.r.t. the true $R$ is measured by the sum of the absolute values of the three Euler angles of the relative rotation $R_{i} R^{T}$. Fig. 2 shows the relative errors w.r.t. noise level. We also check the failure rate defined as the percentage of total trials where either the rotation error or the translation error is over 0.5 (Fig. 3).
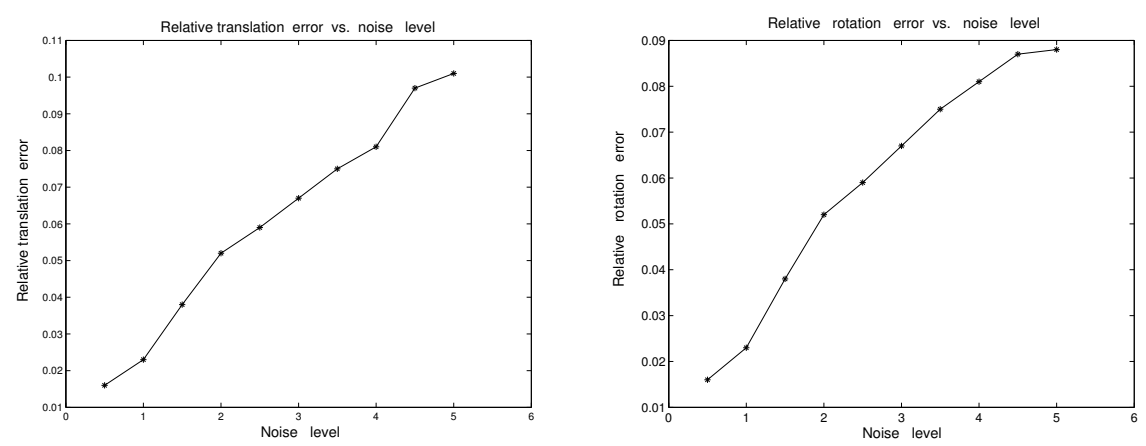

Fig. 2 Relative errors vs. noise level
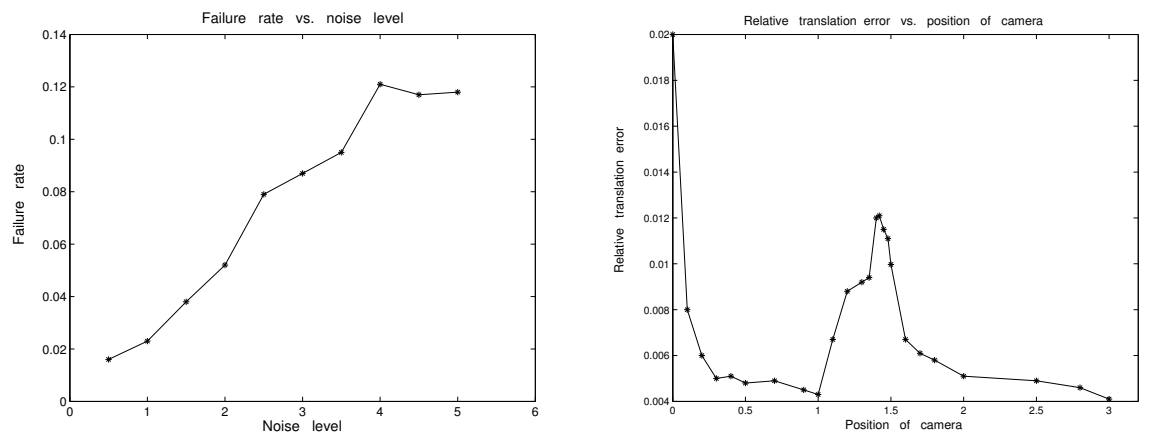
Fig. 3 Failure rate vs. noise level

Fig. 4. Relative translation errors vs. noise level for the critical configurations

The second experiment is to show the accuracy and the stability of the algorithm in determining the solutions for the critical configurations. As mentioned in the introduction, the pose problem has some computationally troublesome singular cases. Fig. 4 and Fig. 5 show the relative error and the failure rate for one such critical configuration using our symbolic-numeric linear method. The data is 4 coplanar points in a square $[-1,1] \times[-1,1]$ and the camera starts at position $=0$, at a singular point directly above their center $(0.5<$ $h<1.5$ ), where $h$ is the height of the camera. The camera then moves sideways parallel to one edge of the square. At position $=\sqrt{2}$ units it crosses the side of the vertical circular cylinder through the 4 data points, where another singularity occurs. From Fig. 4 and Fig.5, the relative error and especially the failure rate of the algorithm are significantly lower compared with the algorithm in [2]. The relative error and the failure rate of our algorithm are also acceptable. It is natural that the error and failure rate near the position 0 and $\sqrt{2}$ are a little higher than at other positions.
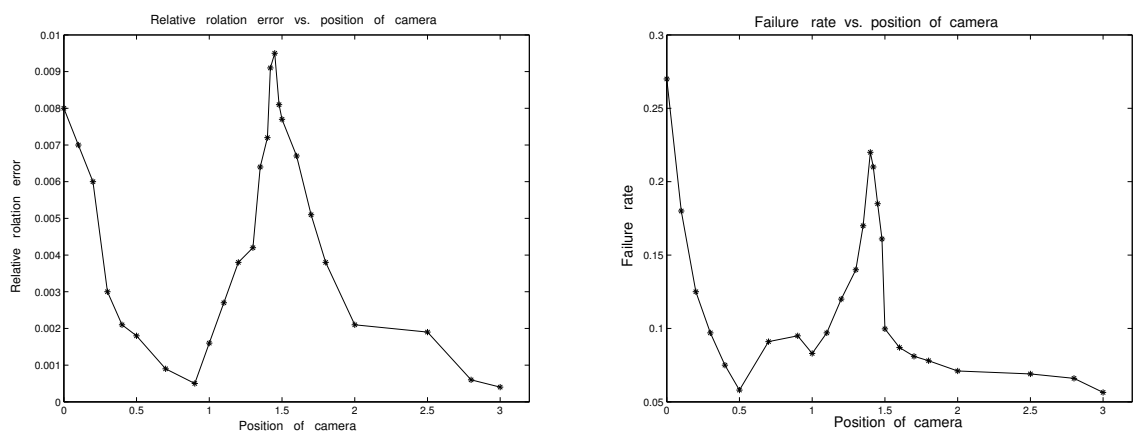

Fig. 5 Relative translation errors vs. noise level for the critical configurations and experimental results for the critical configurations

It is clear that the experimental results are very similar to those we have presented in [23]. However, the computation is simpler due to smaller size of the polynomial system.

\section{Conclusion}

In this paper, we present a stable algorithm to find the numeric solution for 4-point pose estimation. The algorithm gives a unique solution whenever the control points are not sitting on one of the known critical configurations. When the control points are sitting on or near some known critical configurations, the algorithm also obtains reliable solutions. Compared with other algorithms, the main advantage of our linear algorithm is that it can recognize the critical and near critical cases and deal with different cases in different ways. The matrices in our approach are only bigger than those used in other approaches when the points are near critical configurations. The experiments show that the new simple polynomial system for 4-point pose estimation is well solvable by our symbolic-numeric method.

\section{Acknowledgments}


The authors are grateful to X.S. Gao, D.M. Wang for valuable discussions and to MarcAndré Ameller, Bill Triggs and Long Quan for sending us their experimental data.

\section{References}

[1] Abidi, M. A., Chandra, T., A New Efficient and Direct Solution for Pose Estimation Using Quadrangular Targets: Algorithm and Evaluation, IEEE Transaction on Pattern Analysis and Machine Intelligence, 17(5),534-538, 1995.

[2] Ameller, M.A., Triggs, B., and Quan, L., Camera Pose Revisited - New Linear Algorithms, ECCV'00, 2000.

[3] Bonasia, J., Reid, G.J., Zhi, L.H., Determination of approximate symmetries of differential equations, In Gomez-Ullate, Winternitz, editor, CRM Proceedings and Lecture Notes, Amer. Math. Soc. 39, 233-249, 2004.

[4] Buchberger, B., An Algorithm for Finding a Basis for the Residue Class Ring of a ZeroDimensional Polynomial Ideal, PhD. Thesis, Univ. of Innsbruck Math. Inst. 1965.

[5] J.C. Faugére, A New Efficient Algorithm for Computing Gröbner Bases without Reduction to Zero(F5), Proc. ISSAC, T. Mora, ed., New York, ACM Press, 75-83, 2002.

[6] Gao, X.S., Hou, X.R., Tang, J.L. and Cheng, H., Complete Solution Classification for the Perspective-Three-Point Problem, IEEE Tran. on Pattern Analysis and Machine Intelligence, 25(8), 534-538, 2003.

[7] X.-S. Gao, J.L. Tang, On the Solution Number of Solutions for the P4P Problem, MathematicsMechanization Research Center Preprints Preprint, 21, 64-76, 2002.

[8] Horaud, R., Conio, B. and Leboulleux, O., An Analytic Solution for the Perspective 4-Point Problem. CVGIP 47, 33-44, 1989.

[9] Horn, B.K.P., Closed Form Solution of Absolute Orientation Using Unit Quaternions, Journal of the Optical Society of America, 5(7), 1127-1135, 1987.

[10] M. Kuranishi, On E. Cartan's Prolongation Theorem of Exterior Differential Systems, Amer. J. Math, 79, 1-47, 1957.

[11] Z.Y. Hu and F.C. Wu, A Note on the Number Solution of the Non-coplanar P4P Problem, IEEE Transaction on Pattern Analysis and Machine Intelligence, 24(4), 550-555, April 2002.

[12] D. Lazard, Gaussian Elimination and Resolution of Systems of Algebraic Equations, Proc. EUROCAL 83, 146-157, 1993.

[13] Macaulay, F.S., The Algebraic Theory of Modular Systems, Cambridge Univ. Press 19 Cambridge tracts in Math. and Math. Physics, 1916.

[14] B. Mourrain, Computing the Isolated Roots by Matrix Methods, J. Symb. Comput., 26, 715-738, 1998.

[15] B. Mourrain and Ph. Trébuchet, Solving Projective Complete Intersection Faster, Proc. ISSAC, C. Traverso, ed., New York, ACM Press, 430-443, 2000.

[16] H.M. Möller, T. Sauer, H-bases for polynomial interpolation and system solving. Advances Comput. Math., to appear.

[17] B. Mourrain, A New Criterion for Normal Form Algorithms. Proc. AAECC, Fossorier, M.Imai, H.Shu Lin and Poli, A., eds., LNCS, 1719, Springer, Berlin, 430-443, 1999.

[18] Ph. Trébuchet, Vers une Résolution Stable et Rapide des Équations Algébriques. Ph.D. Thesis, Université Pierre et Marie Curie, 2002.

[19] J.F. Pommaret, Systems of Partial Differential Equations and Lie Pseudogroups, Gordon and Breach Science Publishers, 1978. 
[20] Quan, L. and Lan, Z., Linear N-Point Camera Pose Determination, IEEE Transaction on PAMI, 21(8), 774-780, 1999.

[21] P. Rives, P. Bouthémy, B. Prasada, and E. Dubois, Recovering the Orientation and the Position of a Rigid Body in Space from a Single View, Technical Report, INRS-Télécommunications, Quebec, Canada, 1981.

[22] Reid, G.J., Lin, P. and Wittkopf, A.D., Differential elimination-completion algorithms for DAE and PDAE, Studies in Applied Mathematics, 106(1), 1-45,2001.

[23] Reid, G.J., Tang, J. and Lihong Zhi, A complete symbolic-numeric linear method for camera pose determination, Proceedings of the 2003 International Symposium on Symbolic and Algebraic Computation, Scotland, ACM Press, 215-223, 2003.

[24] Reid, G.J., Smith, C. and Verschelde, J., Geometric Completion of Differential Systems using Numeric-Symbolic Continuation, SIGSAM Bulletin 36(2), 1-17, 2002.

[25] H.J. Stetter, Numerical Polynomial Algebra, SIAM, 2004.

[26] Auzinger, W., Stetter, H., An Elimination Algorithm for the Computation of All Zeros of a System of Multivariate Polynomial Equations, Numerical Mathematics Proceedings of the International Conference, Singapore, 11-30, 1988.

[27] Seiler, W.M., Analysis and Application of the Formal Theory of Partial Differential Equations, PhD. Thesis, Lancaster University, 1994.

[28] Sommese, A.J., Verschelde, J. and Wampler, C.W., Numerical decomposition of the solution sets of polynomial systems into irreducible components, SIAM J. Numer. Anal. 38(6), 20222046, 2001.

[29] Wang, D., Characteristic Sets and Zero Structures of Polynomial Sets, Preprint RISC-LINZ, 1989.

[30] Wittkopf, A.D. and Reid, G.J., Fast Differential Elimination in C: The CDiffElim Environment, Comp. Phys. Comm. 139(2) 192-217, 2001.

[31] Wu, W. T.,Basic principles of mechanical theorem proving in geometries Volume I: Part of Elementary Geometries, Science Press Beijing(in Chinese) (1984), English Version SpringerVerlag 1995. 